\title{
Modified-Zero-Fourth-Order-Cumulant-Expansion Approximation Method for Investigation of Turbulence with Reacting and Mixing Chemical Elements
}

\author{
M.C.Meshram ${ }^{1}$ and Kirti Sahu ${ }^{2}$ \\ ${ }^{I}$ Department of Mathematics, Laxminarayan Institute of Technology, Rashtrasant Tukadoji Maharaj Nagpur \\ University, Nagpur-440033(India) \\ ${ }^{2}$ Department of Mathematics, St. Vincent Pallotti College of Engineering and Technology, Nagpur-441108 \\ (India)
}

\begin{abstract}
ABSTRAC: The governing equations for the description of turbulence with reacting and mixing $n$-chemical elements described by R. S. Brodkey ${ }^{1}$ and E. E. O'Brien ${ }^{2}$ are considered. For convenience, we consider a turbulent reaction mixture with two reacting and mixing chemical elements $A$ and $B$. The reactions are irreversible, isothermic, second order and the type of reactions is $A+B \rightarrow$ Product. A brief account of derivation of the equations describing the dynamics of the energy spectrum density functions for the velocity field and the concentration fields $A$ and $B$ derived on using Lewis-Kraichnan ${ }^{3}$ space-time version of the Hopf ${ }^{4}$ functional formalism and multiple-scale-cumulant-expansion method is given. The equations for spectrum functions of the velocity field and the concentration fields derived on employing multiple-scale-cumulant-expansion method by Joshi N. E. and Meshram M. C. ${ }^{5}$ are considered. The equations for spectrum functions are rewritten by using a modified-zero-fourth-order-cumulant-expansion (MZFOCE) approximation method which are first written in dimensionless form and then integrated numerically. The numerical values of the energy of velocity field as well as that of concentration fields are obtained. These values are used to evaluate the statistical quantities describing the turbulence with reacting and mixing chemical elements for large Reynolds numbers up to $R=10^{6}$ .The quantities include energy transfer functions of concentration fields, enstropy of the concentration fields, skewness of concentration fields, dissipation energy of concentration fields and Taylor's micro-scales for concentration fields. We present these in the form of graphs for the representative values of Reynolds number $R=10^{4}$ and $R=10^{6}$. The analysis of the numerical values of statistical parameters thus obtained is carried out and the laws governing these quantities are investigated. Also, we discuss the merits and scope of the present closure scheme for studying similar types of turbulent flows.
\end{abstract}

Key Words: Hopf functional formalism, Cumulants, Multiple scales, Turbulence with reacting and mixing chemical elements

\section{Introduction}

It is accepted that the turbulence itself is viewed as last unsolved problem of classical physics and the addition of the chemical reaction amplifies the difficulties enormously. At first glance, to the uninitiated the subject of turbulent reacting flows would appear to be relatively simple. Indeed, the basic governing principles can be reduced to a statement of conservation of chemical species and energy and a statement of conservation of fluid momentum. However, anyone who has attempted to master this subject will tell you that it is in fact quite complicated subject. On the one hand, in order to understand how the fluid flow affects the chemistry, one must have an excellent understanding of turbulent flows and of turbulent mixing. On the other hand, given its paramount importance in the determination of the types and quantities of chemical species formed, an equally good understanding of chemistry is required. Even a cursory review of the literature in any of these areas will quickly reveal the complexity of the task. Indeed, given the enormous research production in these areas during the twentieth century, it would be safe to conclude that no one could simultaneously master all aspects of turbulence, mixing, and chemistry.

Notwithstanding the intellectual challenges posed by the subject, the main impetus behind the development of computational models for turbulent reacting flows has been the increasing awareness of the impact of such flows on the environment. For example, incomplete combustion of hydrocarbons in internal combustion engines is a major source of air pollution. Likewise, in the chemical process and pharmaceutical industries, inadequate control of product yields and selectivities can produce a host of undesirable by-products. Even if such by-products could all be successfully separated out and treated so that they are not released into the environment, the economic cost of doing so is often prohibitive. Hence, there is an ever-increasing incentive to improve industrial processes and devices in order for them to remain competitive in the marketplace. 
Given their complexity and practical importance, it should be no surprise that different approaches for dealing with turbulent reacting flows have developed over the last sixty years. On the one hand, the chemical-reactionengineering (CRE) approach came from the application of chemical kinetics to the study of chemical reactor design. In this approach, the details of the fluid flow are of interest only in as much as they affect the product yield and selectivity of the reactor. In many cases, this effect is of secondary importance, and thus in the CRE approach greater attention has been paid to other factors that directly affect the chemistry. On the other hand, the fluid-mechanical (FM) approach developed as a natural extension of the statistical description of turbulent flows. In this approach, the emphasis has been primarily on how the fluid flow affects the rate of chemical reactions.

In particular, this approach has been widely employed in the study of combustion. A brief list of such contribution include Rosner ${ }^{6}$ (2000); Peters ${ }^{7}$ (2000); Poinsot and Veynante ${ }^{8}$ (2001); Veynante and Vervisch ${ }^{9}$ (2002), Meshram and Junghare ${ }^{10,11}$ (2004), Meshram ${ }^{12,13}(2010,2012)$, Meshram and Sahu ${ }^{14}$ (2012). In hindsight, the primary factor in determining which approach is most applicable to a particular reacting flow is the characteristic time scales of the chemical reactions relative to the turbulence time scales. In the early applications of the CRE approach, the chemical time scales were larger than the turbulence time scales. In this case, one can safely ignore the details of the flow. Likewise, in early applications of the FM approach to combustion, all chemical time scales were assumed to be much smaller than the turbulence time scales. In this case, the details of the chemical kinetics are of no importance, and one is free to concentrate on how the heat released by the reactions interacts with the turbulent flow. More recently, the shortcomings of each of these approaches have become apparent when applied to systems wherein some of the chemical time scales overlap with the turbulence time scales. In this case, an accurate description of both the turbulent flow and the chemistry is required to predict product yields and selectivities accurately.

With these observations in mind, Joshi N.E. and Meshram M.C. ${ }^{5}$ employed the multiple-scalecumulant-expansion method for the investigation of turbulent reaction mixture with second order irreversible, isothermic reactions for a reaction of type $\mathrm{A}+\mathrm{B} \rightarrow$ Product. We have noticed that the multiple-scale-cumulantexpansion method for chemical reactants enables us to understand their role to get an idea about analysis of various statistical quantities. Such an analysis seems to stimulate a new development in the study of the physical mechanism of turbulence with reacting and mixing chemical elements. There remains, however, an important question concerning the physical basis and the mathematical accuracy of the present approximation at large Reynolds number. Frisch U., Leusieur M. and Schertzer D. ${ }^{15}$ have pointed out that the results obtained on employing multiple-scale-cumulant expansion method by Tatsumi T., Kida S. and Mizushima J. ${ }^{16}$ do not completely describe the statistical features of incompressible isotropic turbulence at large Reynolds numbers. Their observation was based on results they obtained by numerical computations. T. Tatsumi and S. Kida ${ }^{17}$ introduced the modified-zero-fourth-order-cumulant expansion approximation method for isotropic turbulence in response to the defects pointed out by Frisch U., Leusieur M. and Schertzer D. ${ }^{15}$. By introduction of this method for incompressible isotropic turbulence they successfully rectified the defects of earlier method. The method introduced is capable to completely describe the statistical features of incompressible isotropic turbulence for Reynolds numbers as large as $10^{6}$. On extension of the multiple-scale-cumulant expansion method of Tatsumi T., Kida S. and Mizushima J. ${ }^{16}$ to turbulence with reacting and mixing chemical elements of the type A+B $\rightarrow$ Product we could carry out our investigations for Reynolds number up to 1600.This suggests the improvement in the closure scheme. Hence, our maiden natural choice to improve the findings is to employ the modified-zero-fourth-order-cumulant-expansion method for the investigation of turbulence with reacting and mixing chemical elements of the type A+B $\rightarrow$ Product. Joshi N. E. and Meshram M. C. ${ }^{5}$ obtained the dynamical equations for spectrum functions of the velocity fields and the concentration fields on employing multiple-scalecumulant-expansion method. A brief account of these equations is presented in section 2. In section 3 the equations for energy spectrum functions on using modified-zero-fourth-order-cumulant-expansion method are given. Equations for energy spectrum functions in dimensionless form are given in section 4. These equations are integrated numerically and the statistical quantities describing the turbulent reaction mixture with second order reaction are evaluated and detail analysis is carried out in section 5. The presentation concludes with section 6 where the summary of the work is given and results obtained are discussed. This is followed by scope of the method for studying the similar turbulent flows.

\section{Equations For Spectrum Functions Of Velocity Field And Concentration Fields}

The theory of turbulence with reacting and mixing chemical elements with a second order, irreversible , isothermic reactions may be developed in terms of joint probability distribution $\mathrm{P}$ in the space of the vector field $U(\mathrm{x}, t)$ and the $n$-concentration scalar fields $\mathrm{A}_{\propto}(\mathrm{x}, t), \propto=1,2,3, \ldots, n$. which satisfy the following system of equations: 


$$
\begin{aligned}
& \frac{\partial \mathrm{U}_{\mathrm{i}}}{\partial \mathrm{t}}+\mathrm{U}_{\mathrm{j}} \frac{\partial \mathrm{U}_{\mathrm{i}}}{\partial \mathrm{x}_{\mathrm{j}}}=-\frac{1}{\rho} \frac{\partial \mathrm{P}}{\partial \mathrm{x}_{\mathrm{i}}}+\gamma \frac{\partial^{2} \mathrm{U}_{\mathrm{i}}}{\partial \mathrm{x}_{\mathrm{j}} \partial \mathrm{x}_{\mathrm{j}}} \\
& \frac{\partial \mathrm{A}_{\mathrm{P}}}{\partial \mathrm{t}}+\mathrm{U}_{\mathrm{i}} \frac{\partial \mathrm{A}_{\mathrm{P}}}{\partial \mathrm{x}_{\mathrm{i}}}=\mathrm{D} \frac{\partial^{2} \mathrm{~A}_{\mathrm{P}}}{\partial \mathrm{x}_{\mathrm{i}} \partial \mathrm{x}_{\mathrm{i}}}-\mathrm{KA}_{\mathrm{P}} \mathrm{A}_{\mathrm{q}} \\
& \nabla \cdot \mathrm{U}=0
\end{aligned}
$$

Where $U=U(\mathrm{x}, t)=$ velocity of the flow field, $A_{p}=A_{p}(\mathrm{x}, t)=$ concentration of reactant $p, D=$ molecular diffusivity, $\gamma=$ kinematic viscosity and $K=$ reaction rate.

In order to isolate the rate of small-scale fluctuation from other effects, the statistical homogeneity of the concentration fields $A p$ is assumed. Similarly, the turbulence is assumed to be isotropic, homogeneous and axisymmetric. We ignore the dynamic and chemical role of the product. To ensure simplicity, the reaction rate $K$ is selected as constant and the stoichiometric coefficients q as unity. Even though the theory can be developed with these equations for $n$-concentration fields, we restrict ourselves to two reactants A and B keeping future convenience in mind. Similar computations can be conducted for a practical study involving more than two reactants. Thus, for a turbulent mixture with reactants A and B, the system of simultaneous equations (2.1), (2.2) and (2.3) are represented by following equations:

$$
\begin{aligned}
& \frac{\partial \mathrm{U}_{\mathrm{i}}}{\partial \mathrm{t}}+\mathrm{U}_{\mathrm{j}} \frac{\partial \mathrm{U}_{\mathrm{i}}}{\partial \mathrm{x}_{\mathrm{j}}}=-\frac{1}{\rho} \frac{\partial \mathrm{P}}{\partial \mathrm{x}_{\mathrm{i}}}+\gamma \frac{\partial^{2} \mathrm{U}_{\mathrm{i}}}{\partial \mathrm{x}_{\mathrm{j}} \partial \mathrm{x}_{\mathrm{j}}} \\
& \frac{\partial \mathrm{A}}{\partial \mathrm{t}}+\mathrm{U}_{\mathrm{i}} \frac{\partial \mathrm{A}}{\partial \mathrm{x}_{\mathrm{i}}}=\mathrm{D} \frac{\partial^{2} \mathrm{~A}}{\partial \mathrm{x}_{\mathrm{i}} \partial \mathrm{x}_{\mathrm{i}}}-\mathrm{KAB} \\
& \frac{\partial \mathrm{B}}{\partial \mathrm{t}}+\mathrm{U}_{\mathrm{i}} \frac{\partial \mathrm{B}}{\partial \mathrm{x}_{\mathrm{i}}}=\mathrm{D} \frac{\partial^{2} \mathrm{~B}}{\partial \mathrm{x}_{\mathrm{i}} \partial \mathrm{x}_{\mathrm{i}}}-\mathrm{KAB} \\
& \frac{\partial \mathrm{U}_{\mathrm{i}}}{\partial \mathrm{x}_{\mathrm{i}}}=0
\end{aligned}
$$

Here repeated lower indices imply summation. The above equations are described in the work of Brodkey R.S. ${ }^{1}$ and O'Brien E.E. ${ }^{2}$.

The governing equations for description of turbulent mixture with reactants A and B for an isotropic, homogeneous and axisymmetric turbulence are then transformed into a Fourier space by Joshi N.E. and Meshram M.C. ${ }^{5}$. They have defined the characteristic functional for joint probability distribution of velocity and concentration fields. They derived the equations for turbulence under study to the functional differential equations and thereafter, they defined the second characteristic functional. They translated the equations in characteristic functional in terms of second characteristic functional and obtained the equations for the cumulants of various order. These equations describe the dynamics of various order cumulants and also reflect the characteristic difficulty of the turbulence theory that demands a hypothesis to obtain a close set of equations for cumulants. By considering the following hypothesis of multiple scales respectively for length and time as

$$
\begin{gathered}
k_{n}=D^{n / 2} k, n=1,2,3, \ldots \ldots \ldots \\
t_{n}=D^{-n} t, n=0,1,2,3, \ldots \ldots \ldots
\end{gathered}
$$

they successfully obtained a close set of equations for cumulants without any adhoc assumption. The assumption of multiple-scales for the turbulence itself serves the desired purpose.

Even though the equations for higher order cumulant are available they considered equations for second order cumulants and third order cumulant which also involves the fourth order cumulant. On application of multiplescale-cumulant-expansion method they obtained a close set of equation for second order cumulant, since second order cumulants are related to spectral density functions. They derived the following equations describing the 
dynamics of spectral density function for velocity field $\phi^{V}(k, t)$, spectral density function for the reactant A, $\phi^{A}(k, t)$ and the spectral density function for reactant $\mathrm{B}, \phi^{B}(k, t)$.

$$
\begin{gathered}
\left(\frac{\partial}{\partial t}+2 \vartheta k^{2}\right) \phi^{V}(k, t)=4 \pi \int_{-\infty}^{\infty} d k^{\prime} \int_{-1}^{1} \frac{1-e^{-\vartheta\left(k^{2}+k^{\prime 2}+k^{\prime 2}\right) t}}{\vartheta\left(k^{2}+k^{2}+k^{\prime 2}\right)} k k^{\prime 3}\left(\frac{k k^{\prime}}{k^{\prime \prime 2}}+\mu\right)\left(1-\mu^{2}\right) \\
\left\{\left(\phi^{V}\left(k^{\prime}, t\right)-\phi^{V}(k, t)\right) \phi^{V}\left(k^{\prime \prime}, t\right)\right\} d \mu \\
\left(\frac{\partial}{\partial t}+D k^{2}\right) \phi^{A}(k, t)=4 \pi \int_{-\infty}^{\infty} d k^{\prime} \int_{-1}^{1} \frac{1-e^{-D\left(k^{2}+k^{\prime 2}+k^{\prime \prime 2}\right) t}}{D\left(k^{2}+k^{\prime 2}+k^{\prime 2}\right)^{2}} q^{2}\left\{\left(\phi^{V}\left(k^{\prime}, t\right)+\phi^{B}(k, t)\right)\right.
\end{gathered}
$$

$$
\begin{gathered}
\left.\phi^{A}\left(k^{\prime \prime}, t\right)\right\} k k^{\prime 3}\left(\frac{k k^{\prime}}{k^{\prime \prime 2}}+\mu\right)\left(1-\mu^{2}\right) d \mu \\
\left(\frac{\partial}{\partial t}+D k^{2}\right) \phi^{B}(k, t)=4 \pi \int_{-\infty}^{\infty} d k^{\prime} \int_{-1}^{1} \frac{1-e^{-D\left(k^{2}+k^{\prime 2}+k^{\prime \prime 2}\right) t}}{D\left(k^{2}+k^{\prime 2}+k^{\prime 2}\right)} q^{2}\left\{\left(\phi^{V}\left(k^{\prime}, t\right)+\phi^{A}(k, t)\right)\right. \\
\left.\phi^{B}\left(k^{\prime \prime}, t\right)\right\} k k^{\prime 3}\left(\frac{k k^{\prime}}{k^{\prime \prime 2}}+\mu\right)\left(1-\mu^{2}\right) d \mu
\end{gathered}
$$

These equations are integrated numerically by Meshram M. C and Sahu K. ${ }^{18}$.They obtained the statistical quantities describing turbulence with mixing and reacting chemical elements for Reynolds numbers as large as 1600.The results so obtained do not completely describe the statistical features of turbulence at large Reynolds numbers. Hence, we investigate the turbulence with mixing and reacting chemical elements using modified-cumulant-expansion method. This method is described in the section that follows.

\section{Modified-Zero-Fourth-Order-Cumulant-Expansion-Approximation Method}

The numerical results of Frisch U., Leusieur M. and Schertzer D. ${ }^{15}$ reveal that the investigation of homogeneous isotropic turbulence by Tatsumi T., Kida S. and Mizushima J. ${ }^{16}$ on employing multiple-scalecumulant-expansion method do not completely describe the statistical features of incompressible isotropic turbulence at large Reynolds numbers. To examine the situation and consider the problem raised by Frisch U., Leusieur M. and Schertzer D. ${ }^{15}$; Tatsumi T., Kida S. and Mizushima J. ${ }^{16}$ employed modified-zero-fourth-ordercumulant-expansion-approximation method. They got success in describing the statistical features of incompressible isotropic turbulence at large Reynolds number up to $10^{6}$. Hence, we find it worthy to introduce modified-zero-fourth-order-cumulant-expansion-approximation method to study the statistical structure of turbulence with reacting and mixing chemical elements. In the present work we investigated turbulence with reacting and mixing chemical elements for Reynolds number up to $10^{6}$. However, we prefer to present the results which are representative for $R=10^{4}$ and $R=10^{6}$. These results reveal several features of turbulence at these Reynolds numbers which were not clearly detected by using multiple-scale-cumulantexpansion method in earlier investigation.

The equations for energy spectrum function of the velocity field, energy spectrum function of reactant $\mathrm{A}$ and energy spectrum function of reactant B of turbulence with reacting and mixing chemical elements in symbolic form can be written as: 


$$
\begin{aligned}
& \left(\frac{\partial}{\partial t}+\gamma k^{2}\right) E^{V}(k, t)=T^{V}(k, t) \\
& \left(\frac{\partial}{\partial \mathrm{t}}+\gamma \mathrm{k}^{2}\right) \mathrm{E}^{\mathrm{A}}(\mathrm{k}, \mathrm{t})=\mathrm{T}^{\mathrm{A}}(\mathrm{k}, \mathrm{t}) \\
& \left(\frac{\partial}{\partial \mathrm{t}}+\gamma \mathrm{k}^{2}\right) \mathrm{E}^{\mathrm{B}}(\mathrm{k}, \mathrm{t})=\mathrm{T}^{\mathrm{B}}(\mathrm{k}, \mathrm{t})
\end{aligned}
$$

where $\mathrm{E}^{\mathrm{V}}(\mathrm{k}, \mathrm{t}), \mathrm{E}^{\mathrm{A}}(\mathrm{k}, \mathrm{t}), \mathrm{E}^{\mathrm{B}}(\mathrm{k}, \mathrm{t})$ and $\mathrm{T}^{\mathrm{V}}(\mathrm{k}, \mathrm{t}), \mathrm{T}^{\mathrm{A}}(\mathrm{k}, \mathrm{t}), \mathrm{T}^{\mathrm{B}}(\mathrm{k}, \mathrm{t})$ are energy spectrum function for velocity field, energy spectrum function of reactant A, energy spectrum function of reactant $\mathrm{B}$ and energy transfer function for velocity field, energy transfer function of reactant A, energy transfer function of reactant $\mathrm{B}$ respectively, $\mathrm{k}$ is the wavenumber, $\mathrm{t}$ is the time.

Under the modified-zero-fourth-order-cumulant-expansion-approximation method the energy transfer function of velocity field, energy transfer function of reactant A and energy transfer function of reactant B are expressed in terms of the respective energy spectrum function of velocity field, energy spectrum function of reactant $\mathrm{A}$ and energy spectrum function of reactant $\mathrm{B}$ as follows:

$$
\begin{gathered}
T^{V}(k, t)=\int_{0}^{t} d t^{\prime} \int_{0}^{\infty} d k^{\prime} \int_{-1}^{1} e^{-\gamma\left(k^{2}+k^{\prime 2}+k^{\prime 2}\right)\left(t-t^{\prime}\right)} \frac{k k^{\prime}}{k^{\prime \prime 2}}\left(\frac{k k^{\prime}}{k^{\prime \prime 2}}+\mu\right)\left(1-\mu^{2}\right) \\
\left\{\left(k^{2} E^{V}\left(k^{\prime}, t\right)-k^{\prime 2} E^{V}(k, t)\right) E^{V}\left(k^{\prime \prime}, t\right)\right\} d \mu \\
T^{A}(k, t)=\int_{0}^{t} d t^{\prime} \int_{0}^{\infty} d k^{\prime} \int_{-1}^{1} e^{-\gamma\left(k^{2}+k^{\prime 2}+k^{\prime \prime}\right)\left(t-t^{\prime}\right)} \frac{k k^{\prime}}{k^{\prime \prime 2}}\left(\frac{k k^{\prime}}{k^{\prime \prime 2}}+\mu\right)\left(1-\mu^{2}\right) \\
\left\{\left(k^{2} E^{V}\left(k^{\prime}, t\right)-k^{\prime 2} E^{B}(k, t)\right) E^{A}\left(k^{\prime \prime}, t\right)\right\} d \mu \\
T^{\mathbf{B}}(k, t)=\int_{0}^{t} d t^{\prime} \int_{0}^{\infty} d k^{\prime} \int_{-1}^{1} e^{-\gamma\left(k^{2}+k^{\prime 2}+k^{\prime \prime 2}\right)\left(t-t^{\prime}\right)} \frac{k k^{\prime}}{k^{\prime \prime 2}}\left(\frac{k k^{\prime}}{k^{\prime \prime 2}}+\mu\right)\left(1-\mu^{2}\right) \\
\left\{\left(k^{2} E^{V}\left(k^{\prime}, t\right)-k^{\prime 2} E^{A}(k, t)\right) E^{B}\left(k^{\prime \prime}, t\right)\right\} d \mu
\end{gathered}
$$

where $\mathrm{k}^{2}=\mathrm{k}^{2}+\mathrm{k}^{2}+2 \mu \mathrm{k} \mathrm{k}^{\prime}$ and the initial conditions $T^{V}(k, 0)=0, T^{A}(k, 0)=0$

and $T^{B}(k, 0)=0$ have been assumed for simplicity. The set of equation (3.4), (3.5) and (3.6) constitutes a close set of equations for the energy spectrum function of velocity field, energy spectrum function of reactant $\mathrm{A}$ and energy spectrum function of reactant B. Equations for energy spectrum function of velocity field, $E^{V}(k, t)$; energy spectrum function of reactant $\mathrm{A}, E^{A}(k, t)$ and energy spectrum function of reactant $\mathrm{B}, E^{B}(k, t)$ using modified-zero-fourth-order-cumulant-expansion-approximation method become as follows:

$$
\begin{gathered}
\left(\frac{\partial}{\partial t}+\gamma k^{2}\right) E^{V}(k, t)=\int_{-\infty}^{\infty} d k^{\prime} \int_{-1}^{1} \frac{1-e^{-\gamma\left(k^{2}+k^{\prime 2}+k^{\prime 2}\right) t}}{\gamma\left(k^{2}+k^{\prime 2}+k^{\prime 2}\right)} \frac{k k^{\prime}}{k^{\prime \prime 2}}\left(\frac{k k^{\prime}}{k^{\prime \prime 2}}+\mu\right)\left(1-\mu^{2}\right) \\
\left\{\left(k^{2} E^{V}\left(k^{\prime}, t\right)-k^{\prime 2} E^{V}(k, t)\right) E^{V}\left(k^{\prime \prime}, t\right)\right\} d \mu
\end{gathered}
$$




$$
\begin{gathered}
\left(\frac{\partial}{\partial t}+\gamma k^{2}\right) E^{A}(k, t)=\int_{-\infty}^{\infty} d k^{\prime} \int_{-1}^{1} \frac{1-e^{-\gamma\left(k^{2}+k^{\prime 2}+k^{\prime \prime 2}\right) t}}{\gamma\left(k^{2}+k^{\prime 2}+k^{\prime 2}\right)} \frac{k k^{\prime}}{k^{\prime \prime 2}}\left(\frac{k k^{\prime}}{k^{\prime \prime 2}}+\mu\right)\left(1-\mu^{2}\right) \\
\left\{\left(k^{2} E^{V}\left(k^{\prime}, t\right)-k^{\prime 2} E^{B}(k, t)\right) E^{A}\left(k^{\prime \prime}, t\right)\right\} d \mu \\
\left(\frac{\partial}{\partial t}+\gamma k^{2}\right) E^{B}(k, t)=\int_{-\infty}^{\infty} d k^{\prime} \int_{-1}^{1} \frac{1-e^{-\gamma\left(k^{2}+k^{\prime 2}+k^{\prime 2}\right) t}}{\gamma\left(k^{2}+k^{\prime 2}+k^{\prime \prime 2}\right)} \frac{k k^{\prime}}{k^{\prime \prime 2}}\left(\frac{k k^{\prime}}{k^{\prime \prime 2}}+\mu\right)\left(1-\mu^{2}\right) \\
\left\{\left(k^{2} E^{V}\left(k^{\prime}, t\right)-k^{\prime 2} E^{A}(k, t)\right) E^{B}\left(k^{\prime \prime}, t\right)\right\} d \mu \\
\text { where } k^{\prime \prime 2}=k^{2}+k^{\prime 2}+2 \mu k k^{\prime}
\end{gathered}
$$

IV. Equations For Energy Spectrum Functions In Dimensionless Form

For the future convenience of numerical computations all variables are made non-dimensional with respect to a representative value of the energy spectrum $E_{0}$ and a representative value of the wave number $k_{0}$, as functions of the non-dimensional wave number, time and Reynolds number which respectively are given as follows :

$$
\begin{array}{lc}
\text { Wave number } & \kappa=k / k_{0} ; \\
\text { Time } & \tau=E_{0}^{\frac{1}{2}} k_{0}^{\frac{3}{2}} t ; \\
\text { Reynolds number } & R=E_{0}^{\frac{1}{2}} / v k_{0}^{\frac{1}{2}} ;
\end{array}
$$

Thus, the equation (3.7) for energy spectrum function for velocity field $E^{V}(k, t)$, the equation (3.8) for energy spectrum function for the reactant $\mathrm{A}, E^{A}(k, t)$ and the equation (3.9) for the energy spectrum function for reactant $\mathrm{B}, E^{B}(k, t)$ are respectively written in the non-dimensional form as follows:

$$
\begin{gathered}
\left(\frac{\partial}{\partial \tau}+\frac{\kappa^{2}}{R}\right) E^{V}(\kappa, \tau)=R \int_{-\infty}^{\infty} d \kappa^{\prime} \int_{-1}^{1} \frac{1-e^{-\left(\kappa^{2}+\kappa^{\prime 2}+\kappa^{\prime \prime 2}\right) \tau / R}}{\left(\kappa^{2}+\kappa^{\prime 2}+\kappa^{\prime \prime 2}\right)} \frac{\kappa \kappa^{\prime}}{\kappa^{\prime \prime 2}}\left(\frac{\kappa \kappa^{\prime}}{\kappa^{\prime \prime 2}}+\mu\right)\left(1-\mu^{2}\right) \\
\left\{\left(\kappa^{2} E^{V}\left(\kappa^{\prime}, \tau\right)-\kappa^{\prime 2} E^{V}(\kappa, \tau)\right) E^{V}\left(\kappa^{\prime \prime}, \tau\right)\right. \\
\left(\frac{\partial}{\partial \tau}+\frac{\kappa^{2}}{R}\right) E^{A}(\kappa, \tau)=R \int_{-\infty}^{\infty} d \kappa^{\prime} \int_{-1}^{1} \frac{1-e^{-\left(\kappa^{2}+\kappa^{\prime 2}+\kappa^{\prime \prime 2}\right) \tau / R}}{\left(\kappa^{2}+\kappa^{\prime 2}+\kappa^{\prime \prime 2}\right)} \frac{\kappa \kappa^{\prime}}{\kappa^{\prime \prime 2}}\left(\frac{\kappa \kappa^{\prime}}{\kappa^{\prime \prime 2}}+\mu\right)\left(1-\mu^{2}\right) \\
\left\{\left(\kappa^{2} E^{V}\left(\kappa^{\prime}, \tau\right)-\kappa^{\prime 2} E^{B}(\kappa, \tau)\right) E^{A}\left(\kappa^{\prime \prime}, \tau\right)\right. \\
\left(\frac{\partial}{\partial \tau}+\frac{\kappa^{2}}{R}\right) E^{B}(\kappa, \tau)=R \int_{-\infty}^{\infty} d \kappa^{\prime} \int_{-1}^{1} \frac{1-e^{-\left(\kappa^{2}+\kappa^{\prime 2}+\kappa^{\prime \prime 2}\right) \tau / R}}{\left(\kappa^{2}+\kappa^{\prime 2}+\kappa^{\prime 2}\right)} \frac{\kappa \kappa^{\prime}}{\kappa^{\prime \prime 2}}\left(\frac{\kappa \kappa^{\prime}}{\kappa^{\prime \prime 2}}+\mu\right)\left(1-\mu^{2}\right) \\
\left\{\left(\kappa^{2} E^{V}\left(\kappa^{\prime}, \tau\right)-\kappa^{\prime 2} E^{A}(\kappa, \tau)\right) E^{B}\left(\kappa^{\prime \prime}, \tau\right)\right.
\end{gathered}
$$


where $\kappa^{\prime \prime 2}=\kappa^{2}+\kappa^{\prime 2}+2 \mu \kappa \kappa^{\prime}$.

We must emphasize that the present study relates to the investigation of turbulence with reacting and mixing chemical elements A and B. Here A and B are not chosen as any specific reactants as we wish to present the investigations in the general form. For the study of specific reactants their corresponding initial values for spectral density functions may be selected and the investigation be carried out corresponding to those specific reactants. Also, for the present study we have selected Schmidt number $S c\left(S c=\frac{\vartheta}{D}\right.$ ) as unity since not much information is lost by this assumption. The Schmidt number represents the ratio of the kinematic viscosity of the fluid and the molecular diffusivity of particular specie. We have also assumed equal diffusivities for all species. We select the initial conditions for spectral density functions of velocity fields, reactant $\mathrm{A}$ and the reactant $\mathrm{B}$ as follows

$$
\begin{gathered}
\phi^{V}(\kappa, 0)=\frac{1}{4 * p i} \exp \left(-\kappa^{2}\right) \\
\phi^{A}(\kappa, 0)=\frac{1}{40 * p i} \exp \left(-\kappa^{2}\right) \\
\phi^{B}(\kappa, 0)=\frac{1}{400 * p i} \exp \left(-\kappa^{2}\right)
\end{gathered}
$$

Similarly we select the initial conditions for the energy spectrum function for velocity field, energy spectrum function for the reactant $\mathrm{A}, E^{A}(k, t)$ and the energy spectrum function for the reactant $\mathrm{B}$, $E^{B}(k, t)$ as given below by equations (4.5a), (4.5b) and (4.5c) respectively.

$$
\begin{aligned}
& E^{V}(k, 0)=E_{0}^{V}\left(k / k_{0}\right)^{2} \exp \left[-\left(k / k_{0}\right)^{2}\right] \\
& E^{A}(k, 0)=E_{0}^{A}\left(k / k_{0}\right)^{2} \exp \left[-\left(k / k_{0}\right)^{2}\right] \\
& E^{B}(k, 0)=E_{0}^{B}\left(k / k_{0}\right)^{2} \exp \left[-\left(k / k_{0}\right)^{2}\right]
\end{aligned}
$$

The integrals on the right -hand side of equations (4.1) to (4.3) are calculated numerically by using appropriate sum rules at discrete values of $\kappa^{\prime}$ and $\mu$ and terminating the infinite integrals with respect to $\kappa^{\prime}$ at a sufficiently large value of $\kappa^{\prime}$. At very large Reynolds number the integrand changes rapidly at small $\kappa^{\prime}$ and does not vanish even at large $\boldsymbol{\kappa}^{\prime}$. In order to evaluate such an integral accurately and efficiently we employ a non-uniform mesh for $\boldsymbol{K}^{\prime}$ whose size is an increasing function of $\boldsymbol{K}^{\prime}$. To meet this requirement we introduced the following variables $\xi$ and $\eta$ :

$$
\xi=\log (10 \kappa) ; \eta=0.01 R \tau \text { so that } \phi(\xi, \eta)=0.1 \phi(\kappa, \tau)
$$

In terms of these variables equations (4.1), (4.2) and (4.3) takes the form as given below:

$$
\begin{aligned}
& \left(\frac{\partial}{\partial \eta}+\frac{e^{2 \xi}}{R^{2}}\right) E^{V}(\xi, \eta) \\
& =\int_{-\infty}^{\infty} e^{3 \xi^{\prime}} d \xi^{\prime} \int_{-1}^{1}\left(\frac{1-\exp \left[-2 e^{\xi+\xi^{\prime}}\left(e^{\xi-\xi^{\prime}}+e^{\xi^{\prime}-\xi}+\mu\right) \eta / R^{2}\right]}{e^{\xi^{-\xi^{\prime}}}+e^{\xi^{\prime}-\xi}+\mu}\right)\left(\frac{1}{\left.e^{\xi^{-\xi^{\prime}}+e^{\xi^{\prime}-\xi^{\prime}}+2 \mu}+\mu\right)}\right. \\
& \quad\left(1-\mu^{2}\right) \times\left\{\left(e^{2 \xi} E^{V}\left(\xi^{\prime}, \eta\right)-e^{2 \xi^{\prime}} E^{V}(\xi, \eta)\right) E^{V}\left(\xi^{\prime \prime}, \eta\right)\right\} d \mu
\end{aligned}
$$




$$
\begin{aligned}
& \left(\frac{\partial}{\partial \eta}+\frac{e^{2 \xi}}{R^{2}}\right) E^{A}(\xi, \eta) \\
& =\int_{-\infty}^{\infty} e^{3 \xi^{\prime}} d \xi^{\prime} \int_{-1}^{1}\left(\frac{1-\exp \left[-2 e^{\xi+\xi^{\prime}}\left(e^{\xi-\xi^{\prime}}+e^{\xi^{\prime}-\xi}+\mu\right) \eta / R^{2}\right]}{e^{\xi^{-\xi^{\prime}}}+e^{\xi^{\prime}-\xi}+\mu}\right)\left(\frac{1}{\left.e^{\xi^{-\xi^{\prime}}+e^{\xi^{\prime}-\xi}+2 \mu}+\mu\right)\left(1-\mu^{2}\right)}\right. \\
& \times\left\{\left(e^{2 \xi} E^{V}\left(\xi^{\prime}, \eta\right)-e^{2 \xi^{\prime}} E^{B}(\xi, \eta)\right) E^{A}\left(\xi^{\prime \prime}, \eta\right)\right\} d \mu \\
& \left(\frac{\partial}{\partial \eta}+\frac{e^{2 \xi}}{R^{2}}\right) E^{\mathbf{B}}(\xi, \eta) \\
& =\int_{-\infty}^{\infty} e^{3 \xi^{\prime}} d \xi^{\prime} \int_{-1}^{1}\left(\frac{\left.1-\exp \left[-2 e^{\xi+\xi^{\prime}}\left(e^{\xi_{-} \xi^{\prime}}+e^{\xi^{\prime}-\xi}+\mu\right) \eta / R^{2}\right]\right)\left(\frac{1}{e^{\xi-\xi^{\prime}}+e^{\xi^{\prime}-\xi^{\prime}}+\mu}\right)\left(\frac{1}{e^{\xi-\xi^{\prime}}+e^{\xi^{\prime}-\xi}+2 \mu}\right)\left(1-\mu^{2}\right)}{} \times\left\{\left(e^{2 \xi} E^{V}\left(\xi^{\prime}, \eta\right)-e^{2 \xi^{\prime}} E^{A}(\xi, \eta)\right) E^{B}\left(\xi^{\prime \prime}, \eta\right)\right\} d \mu\right.
\end{aligned}
$$

\section{Evaluation Of Statistical Quantities And Investigation Of Governing Laws}

The numerical integration of equations (4.7), (4.8) and (4.9) is carried out for $\mathrm{R}=10^{4}, 10^{5}$ and $10^{6}$ using the same numerical method as employed for multiple-scale-cumulant-expansionmethod but employing the larger cutoff wave number for the $\mathcal{K}^{\prime}$ integral and finer steps for the time integration. The cutoff wavenumber is taken as $\kappa^{\prime}=k^{\prime} / k_{0}=0.1 \times 1.2^{N}$ with

$$
\mathrm{N}=\left\{\begin{array}{ccc}
60 & \text { for } & \mathrm{R}=10^{4} \\
70 & \text { for } & 10^{4}<\mathrm{R} \leq 10^{5} \\
80 & \text { for } & 10^{5}<\mathrm{R} \leq 10^{6}
\end{array}\right.
$$

The time step is taken as $\Delta \tau=0.2$. Thus, the equations (4.7), (4.8) and (4.9) are solved numerically for the initial conditions $(4.5 \mathrm{a})$, (4.5b) and (4.5c); the Reynolds number $\mathrm{R}=10^{4}$ to $\mathrm{R}=10^{6}$; and the energy spectrum functions for reactant $\mathrm{A}, E^{A}(k, t)$ and energy spectrum functions for reactant $\mathrm{B}, E^{B}(k, t)$ are obtained as functions of the wave number $\mathrm{k}$ and time t. Important statistical quantities such as energy of concentration fields $E^{A}(k, t)$ and $E^{B}(k, t)$, transfer functions of concentration fields $T^{A}(k, t)$ and $T^{B}(k, t)$, enstropy of concentration fields $\varepsilon^{A}(t)$ and $\varepsilon^{B}(t)$, skewness of concentration fields $S^{A}(t)$ and $S^{B}(t)$, dissipation energy of concentration fields $D^{A}(k, t)$ and $D^{B}(k, t)$ and Taylor's micro-scales for concentration fields $\lambda^{A}(t)$ and $\lambda^{B}(t)$; characterizing the turbulence with reacting and mixing chemical elements of the type $\mathrm{A}+\mathrm{B} \rightarrow$ Product are derived from numerical values of energy spectrum functions. We prefer to present only representative of these corresponding to $\mathrm{R}=10^{4}$ and $\mathrm{R}=10^{6}$. In the entire discussion below the energy spectrum function $E^{V}(k, t)$ shall not be discussed as all relations governing $E^{V}(k, t)$ are not different from those obtained by T. Tatsumi et.al. ${ }^{17}$ and do not fetch any additional information for the velocity field using modifiedcumulant-expansion method.

Of all the numerical data concerning the evolution of energy spectrum functions $E^{A}(k, t)$ and $E^{B}(k, t)$ of concentration fields $\mathrm{A}$ and $\mathrm{B}$ respectively for $\mathrm{R}=10^{4}$ and $\mathrm{R}=10^{6}$ are chosen as representative data and shown graphically in figure 5.1 and figure 5.2 respectively. T. Tatsumi et.al. ${ }^{17}$ on employing modified-cumulantexpansion-approximation method for the velocity field obtained positive energy spectrum function at all times for all the Reynolds numbers. On extending this method to the turbulence with reacting and mixing chemical 
Modified-Zero-Fourth-Order-Cumulant-Expansion Approximation Method for Investigation of

elements of the type $\mathrm{A}+\mathrm{B} \rightarrow$ Product we also obtained positive energy spectrum function for both concentration fields $\mathrm{A}$ and $\mathrm{B}$ at all times for all the Reynolds numbers as large as $10^{6}$. The spectrum of the concentration fields extends much further into the high wave number range than that of the velocity field.

The form of the spectrum function $E^{A}(k, t)$ and $E^{B}(k, t)$ of the reactant A and B especially its structure at higher wave numbers, are displayed more clearly on logarithmic scale as shown in figure 5.1 and figure 5.2. There are some remarkable features in the evolution of the spectrum functions shown in these figures. First, the positive slopes of $E^{A}(k, t)$ and $E^{B}(k, t)$ at very low wave numbers, which are almost invariant in time. Thus, the spectrum functions in the very low wave number range are entirely fixed by the initial conditions, reflecting the well known permanence of the large scale component of turbulence. Second, in the similarity period the higher wave number part of the spectrum are not influenced appreciably by the difference between the cases corresponding to Reynolds numbers $10^{4}$ and $10^{6}$ at very low wave numbers but has a nearly identical form for both the cases. The more detailed structure of the spectrum in the similarity period is dependent upon the Reynolds number, so that it will be described below for small and large Reynolds numbers in turn. A closer inspection of the spectrum reveals that the dependence of the spectrum functions on the wave number is not precisely that of $\exp \left(-2 \vartheta k^{2} \mathrm{t}\right)$ as would be expected from the linearized form of (4.1),(4.2) and (4.3) and the initial conditions $(4.5 \mathrm{a}),(4.5 \mathrm{~b})$ and $(4.5 \mathrm{c})$ but is more closely represented by

$$
E^{A}(k, t) \propto \exp \left(\sigma k^{1.5}\right)
$$

$$
E^{B}(k, t) \propto \exp \left(\sigma k^{1.5}\right)
$$

as $\mathrm{k} \rightarrow \infty$, where $\boldsymbol{\sigma}$ is a constant dependent upon $\vartheta$ and t. The spectrum functions in the universal wave number range are composed of three parts having different functional forms.

In the lowest subrange of the universal wave number range, the energy spectrum functions follow the laws which are expressed as follows:

$$
\begin{aligned}
& E^{A}(k, t) \propto k^{-\frac{5}{3}} \\
& E^{B}(k, t) \propto k^{-\frac{5}{3}}
\end{aligned}
$$

The energy spectrum functions of reactant $A$ and that of reactant $B$ is proportional to the negative $\frac{5}{3}$

power of the wave number. We notice that the above law for the energy spectrum functions of reactant $A$ and reactant B has exactly the same power as Kolomogorov's inertial-subrange spectrum. This result is in accordance with the Kolomogorov's second hypothesis, which assumes the existence of the inertial subrange at large Reynolds numbers. The part of the subrange in which Kolomogorov's second hypothesis are valid increases indefinitely with Reynolds number. The negative $\frac{5}{3}$ power for spectrum functions have been observed by many experiments in various turbulent flows with extremely large Reynolds number either in nature or in laboratory.

(ii) Next to previous subrange, there exists a subrange in which the spectrum functions follow the laws as follows:

The energy spectrum functions of the reactant $\mathrm{A}, E^{A}(k, t)$ and the reactant $\mathrm{B}, E^{B}(k, t)$ are inversely proportional to a wave number.

Symbolically the laws are written as follows:

$$
E^{A}(k, t) \propto k^{-1}
$$

$$
E^{B}(k, t) \propto k^{-1}
$$

As the Reynolds number increases, this subrange is shifted to higher wave number without changing its width on logarithmic scale but becomes wider on linear scale.

(iii) At still higher wave number, both the spectrum functions $E^{A}(k, t)$ and $E^{B}(k, t)$

decays exponentially.

Thus, the results obtained by the application of modified-cumulant-expansion- approximation method support the asymptotic decay of the spectrum functions for very high wave number irrespective of Reynolds numbers. 
The energy transfer function of the reactant $\mathrm{A}, T^{A}(k, t)$ and of the reactant $\mathrm{B}, T^{B}(k, t)$ respectively those for $\mathrm{R}=10^{4}$ and $\mathrm{R}=10^{6}$ are shown graphically in figure 5.3 and figure 5.4. From these figures we notice that the energy transfer is from lower to higher wave numbers. The nature of the transfer spectra of both the chemical reactants A and B is analogous. The difference observed in the graphs for $T^{A}(k, t)$ and $T^{B}(k, t)$ at different times is mainly due to the difference in the choice of their respective initial values. Both transfer spectra are positive in the first shell.

The present closure scheme employed for investigation leads to the result that, for a given Reynolds number, in the neighbourhood of the wave number both the energy transfer functions change their sign and satisfy the following requirement

$$
\int_{0}^{\infty} T^{A}(k, t) d k=0
$$

$$
\int_{0}^{\infty} T^{B}(k, t) d k=0
$$

We further notice that as the Reynolds number takes the larger value the wavenumber at which $T^{A}(k, t)$ and $T^{B}(k, t)$ vanish is shifted to the lower wavenumber.

The enstropy $\varepsilon^{A}(t)$ of the concentration field A and enstropy $\varepsilon^{B}(t)$ of the concentration field B are respectively defined by the following integrals:

$$
\begin{gathered}
\varepsilon^{A}(t)=4 \pi \int_{0}^{\infty} k^{2} \phi^{A}(k, t) d k \\
\varepsilon^{B}(t)=4 \pi \int_{0}^{\infty} k^{2} \phi^{B}(k, t) d k
\end{gathered}
$$

These quantities mostly reflect the shape of energy spectrum functions $E^{A}(k, t)$ and $E^{B}(k, t)$ in the energy containing range of the wave number.

The equations (4.7), (4.8) and (4.9) are integrated numerically and obtained the numerical values of the energy spectrum functions of the velocity field, energy spectrum functions of the reactant $\mathrm{A}$ and the reactant $B$. The values of energy spectrum functions thus obtained are used to find the numerical values of enstropy of the reactant $\mathrm{A}$ and $\mathrm{B}$ and the plots of these values are shown in figure 5.5 and figure 5.6 respectively. We infer from these graphs of enstropy for reactant A and B that they are analogous to each other. The shape of each of these graphs can be divided into three parts depending upon their behaviour with respect to time. The first part being from $\mathrm{t}=0$ to $\mathrm{t}=60$, the second part from $\mathrm{t}=60$ to $\mathrm{t}=135$ and third part $\mathrm{t}>135$. The enstropy for both the reactants, $\varepsilon^{A}(t)$ and $\varepsilon^{\mathbf{B}}(t)$ increases monotonically with respect to time in the initial period from $\mathrm{t}=0$ to $\mathrm{t}$ $=60$. Thereafter in the second period from $\mathrm{t}=60$ to $\mathrm{t}=135$ the curves attain the Gaussian shape. In the final period corresponding to $t>135$ the enstropy of both the reactants increases monotonically. The difference in enstropy of concentration fields A and concentration field B is solely due to the choice of initial conditions. We also observe from these figures that as the Reynolds number increases there is increase in the time taken to attain the maximum enstropy in case of both the reactants A and B. For both the reactants their enstrophy grows very rapidly in the initial period. The enstropy of the reactant $A$ attains a quite large maximum value 21.5 for $R=10^{4}$ and 17.5 corresponding to $R=10^{6}$. Similarly the corresponding maximum values for the reactant $B$ are 16.5 and 13.5 respectively. This reveals that there is a fall in the maximum value of enstropy with rise in the Reynolds number.

In the initial period before attaining the maximum value the enstropy of the specie $\mathrm{A}$ is proportional to second power of time and analogues to this enstropy of the specie B is proportional to 2.25 power of time. Symbolically these laws are expressed as follows:

$$
\begin{gathered}
\varepsilon^{A}(t) \alpha t^{2} \\
\varepsilon^{B}(t) \alpha t^{2.25}
\end{gathered}
$$


Next to previous period just after attaining the maximum value for $R=10^{4}$ and $R=10^{6}$ the value of enstropy of both the reactants decreases and this decrease is governed by the following laws:

The enstropy of the concentration field A is proportional to the negative 2.1 power of time and the enstropy of the concentration field $\mathrm{B}$ is proportional to the negative 2.13 power of time .

Symbolically these laws are written as follows:

$$
\varepsilon^{A}(t) \alpha t^{-2.1}
$$

$$
\varepsilon^{B}(t) \alpha t^{-2.13}
$$

These laws agree quite well with the experimental results.

In the last period enstropy of the reactant $A$ and that of $B$ varies with respect to time as per the following laws: The enstropy of the concentration field A is proportional to the 1.13 power of time whereas the enstropy of the concentration field B is proportional to the 1.03 power of time. These laws symbolically are written as follows:

$$
\begin{gathered}
\varepsilon^{A}(t) \alpha t^{1.13} \\
\varepsilon^{B}(t) \propto t^{1.03}
\end{gathered}
$$

The skewness of the distribution of the time derivative of the reactant A, $S^{A}(t)$ and reactant B, $S^{B}(t)$ is defined and related to the corresponding energy spectrum functions and the energy transfer functions as follows:

$$
\begin{aligned}
& S^{A}(t)=\frac{3 \sqrt{30}}{14} \frac{\int_{0}^{\infty} k^{2} T^{A}(k, t) d k}{\left[\int_{0}^{\infty} k^{2} E^{A}(k, t) d k\right]^{3 / 2}} \\
& S^{B}(t)=\frac{3 \sqrt{30}}{14} \frac{\int_{0}^{\infty} k^{2} T^{B}(k, t) d k}{\left[\int_{0}^{\infty} k^{2} E^{B}(k, t) d k\right]^{3 / 2}}
\end{aligned}
$$

Equations (5.17) and (5.18) for skewness of the reactant $\mathrm{A}, S^{A}(t)$ and of the reactant B, $S^{B}(t)$ reveal their dependence on energy spectrum functions $E^{A}(k, t), E^{B}(k, t)$ and energy transfer functions $T^{A}(k, t), T^{B}(k, t)$. The skewness of both the reactants is mainly determined by the forms of energy spectrum functions $E^{A}(k, t), E^{B}(k, t)$ and energy transfer functions $T^{A}(k, t), T^{B}(k, t)$ in the higher wavenumber range, so that it reflects the shape of the energy spectrum $E^{A}(k, t), E^{B}(k, t)$ beyond the energy-containing range of the wave numbers. The skewness of both the reactants is evaluated by substituting numerical values of $E^{A}(k, t), E^{B}(k, t)$ and $T^{A}(k, t), T^{B}(k, t)$ into (5.17) and (5.18).

The numerical values of skewness of both the reactants are evaluated and presented in the form of graphs in figures 5.7 for Reynolds number $\mathrm{R}=10^{4}$ and in figure 5.8 for Reynolds number $\mathrm{R}=10^{6}$. The initial value of skeness is identically zero for both the reactants due to our choice of the initial conditions. The chemical reaction has a dramatic effect upon the evolution of the skewness coefficient. The skewness $S^{A}(t)$ and $S^{B}(t)$ defined by (5.17) and (5.18) gives a non-dimensional measure of the strength of the vorticity production. Since $S^{A}(t)$ and $S^{B}(t)$ is expressed as an integral of $E^{A}(k, t)$ and $E^{B}(k, t)$ respectively weighted towards higher wave numbers, it reflects the behaviour of the spectrum in the universal range, or the small-scale structure of turbulence with reaction mixture. Also, from these figures we notice that the skewness of both the reactants is a measure of the departure from Gaussianity and indicate how transfer functions vary 
with the time at high wave number. The skewness of both the reactants decreases substantially after having reached their sharp maximum and then increases monotonically with respect to time. Hence depending upon their behaviour with respect to time the shape of each of these graphs can be divided into three parts. The first part being from $t=0$ to $t=60$, the second part from $t=60$ to $t=135$ and third part $t>135$. The skewness for both the reactants, $S^{A}(t)$ and $S^{\mathbf{B}}(t)$ increase monotonically with respect to time in the initial period from $\mathrm{t}=0$ to $t=60$. Thereafter in the second period from $t=60$ to $t=135$ the curves assumes the Gaussian shape. In the final period corressponding to $t>135$ the skewness of both the reactants increases monotonically. The maximum value of the skewness attained by the reactant $A$ for $R=10^{4}$ is 21.5 and that for the reactant $B$ is nearly 16.5 . Similarly for $\mathrm{R}=10^{6}$ the maximum values of the skewness attained by the reactant A and reactant B respectively are 17.5 and 13.5 approximately. This reveals that there is a fall in the maximum value of skewness with rise in the Reynolds number.

In the initial period before attaining the maximum value the skewness of the specie $\mathrm{A}$ is proportional to second power of time and analogues to this skewness of the specie B is proportional to 2.25 power of time .These laws symbolically are written as follows:

$$
\begin{gathered}
S^{A}(t) \alpha t^{2} \\
S^{B}(t) \alpha t^{2.25}
\end{gathered}
$$

Next to previous period just after attaining the maximum value for $R=10^{4}$ and $R=10^{6}$ the value of skewness of both the reactants decreases and this decrease is governed by the following laws:

The skewness of the concentration field $\mathrm{A}$ is proportional to the negative 2.1 power of time and this law for the skewness of the concentration field B is proportional to the negative 2.13 power of time. These laws symbolically are written as follows:

$$
\begin{gathered}
S^{A}(t) \propto t^{-2.1} \\
S^{B}(t) \propto t^{-2.13}
\end{gathered}
$$

In the last period skewness of the reactant $\mathrm{A}$ and $\mathrm{B}$ varies with respect to time as per the following laws: The skewness of the reactant $\mathrm{A}$ is directly proportional to 1.13 power of time and similarly the skewness of the reactant $\mathrm{B}$ is directly proportional to 1.03 power of time. These laws symbolically are written as follows:

$$
\begin{aligned}
& S^{A}(t) \propto t^{1.13} \\
& S^{B}(t) \propto t^{1 . .03}
\end{aligned}
$$

The energy dissipation function for concentration field A and concentration field B respectively are defined below as:

$$
\begin{aligned}
& D^{A}(k, t)=2 v k^{2} E^{A}(k, t) \\
& D^{B}(k, t)=2 v k^{2} E^{B}(k, t)
\end{aligned}
$$

The numerical values of energy spectrum functions of both the reactants are used to evaluate their dissipation functions. The values so obtained are plotted verses wavenumbers at time $t=0$ and $t=3$ for Reynolds numbers $\mathrm{R}=10^{4}$ in figure 5.9 and $\mathrm{R}=10^{6}$ in figure 5.10. These graphs clearly reflect the characteristic of energy spectra .It is observed that for large Reynolds number, in the energy containing range and the inertial subrange the energy dissipation is much bigger in magnitude than the energy transfer.

Finally it is observed that for large Reynolds number the $\mathrm{k}^{-1}$ spectrum always occurs in the neighbourhood of wavenumber at which energy transfer changes its sign.

$$
\begin{aligned}
& \int_{0}^{\infty} T^{A}(k, t) d k=0 \\
& \int_{0}^{\infty} T^{B}(k, t) d k=0
\end{aligned}
$$

Lastly, the Taylor's micro-scale for the reactants $\mathrm{A}, \lambda^{A}(t)$ and for the reactant $\mathrm{B}, \lambda^{B}(t)$ are defined and expressed in terms of the energy spectrum functions as given below: 


$$
\lambda^{A}(t)=\frac{5 \int_{0}^{\infty} E^{A}(k, t) d k}{\int_{0}^{\infty} k^{2} E^{A}(k, t) d k}
$$

$$
\lambda^{B}(t)=\frac{5 \int_{0}^{\infty} E^{B}(k, t) d k}{\int_{0}^{\infty} k^{2} E^{B}(k, t) d k}
$$

The values of Taylor's micro-scale of reactant $\mathrm{A}, \lambda^{A}(t)$ and that of reactant $\mathrm{B}, \lambda^{B}(t)$ obtained by substituting numerical values of $E^{A}(k, t)$ and $E^{B}(k, t)$ into (5.29) and (5.30) and the numerical values thus obtained are plotted in figure 5.11 for Reynolds number $\mathrm{R}=10^{4}$ and in figure 5.12 for Reynolds number $\mathrm{R}=10^{6}$. The Taylor's micro-scale defined by (5.29) and (5.30) gives another parameter representing the small scale structure of turbulence with reacting and mixing chemical elements A and B. At large Reynolds numbers the micro-scale for the reactant $\mathrm{A}$ and that of the reactant $\mathrm{B}$ decrease very rapidly in the initial stage. Then thereafter for $\mathrm{R}=10^{4}$ the Taylor's micro-scale of reactant $\mathrm{A}$ attains a minimum around $\mathrm{t}=1.2$ and that of the reactant $\mathrm{B}$ attains a minimum around $\mathrm{t}=0.5$ and these values for $\mathrm{R}=10^{6}$ respectively are $\mathrm{t}=0.8$ and $\mathrm{t}=0.4$.

The variation of the Taylor's micro-scale of reactant A and that of the reactant $\mathrm{B}$

with time is governed by the laws that the Taylor's microscale of both the reactants varies proportionally to negative 0.5 power of the time. Symbolically these laws are written as follows:

$$
\begin{aligned}
& \lambda^{A}(t) \propto t^{-0.5} \\
& \quad \lambda^{B}(t) \alpha t^{-0.5}
\end{aligned}
$$

The variation of the Taylor's micro-scale of reactant A and that of the reactant B with time confirms the asymptotic decrease of Taylor's micro-scale with time.
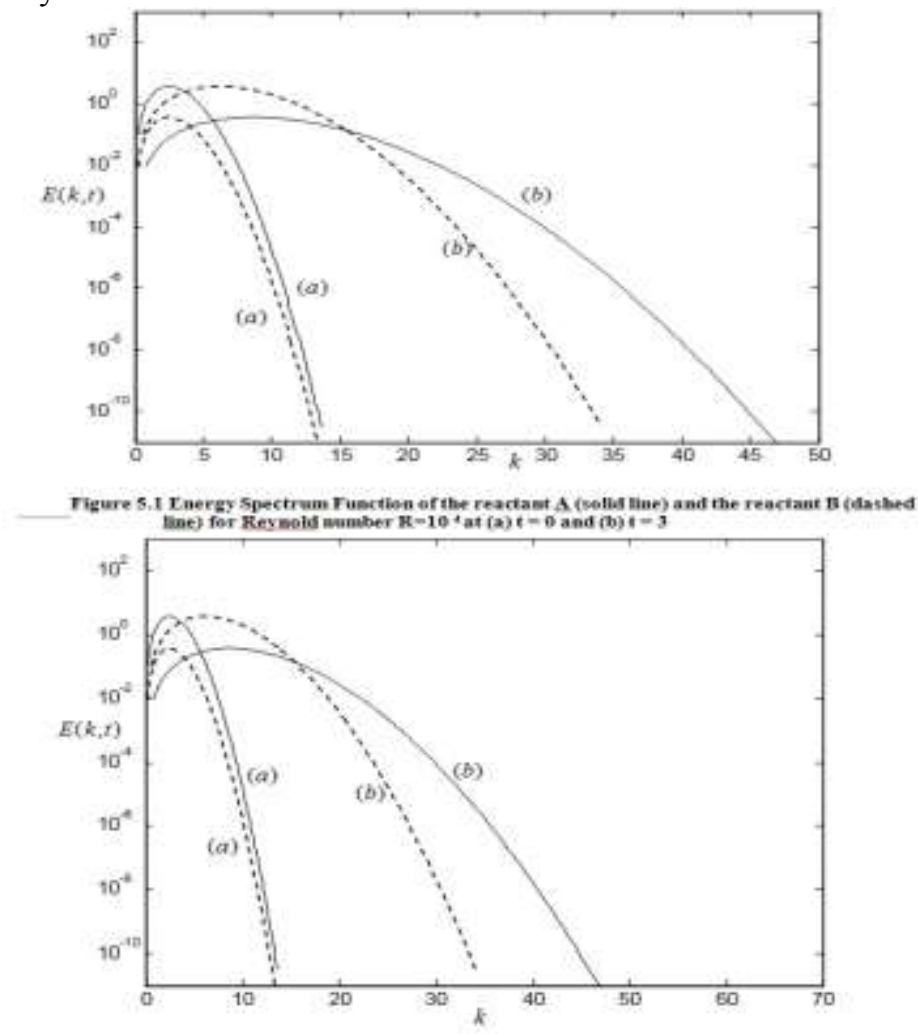

Figure 5,2 Energy Spectrum Functios of the reactant A (solid hine) and tbe reactast B (dashed

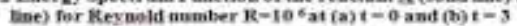




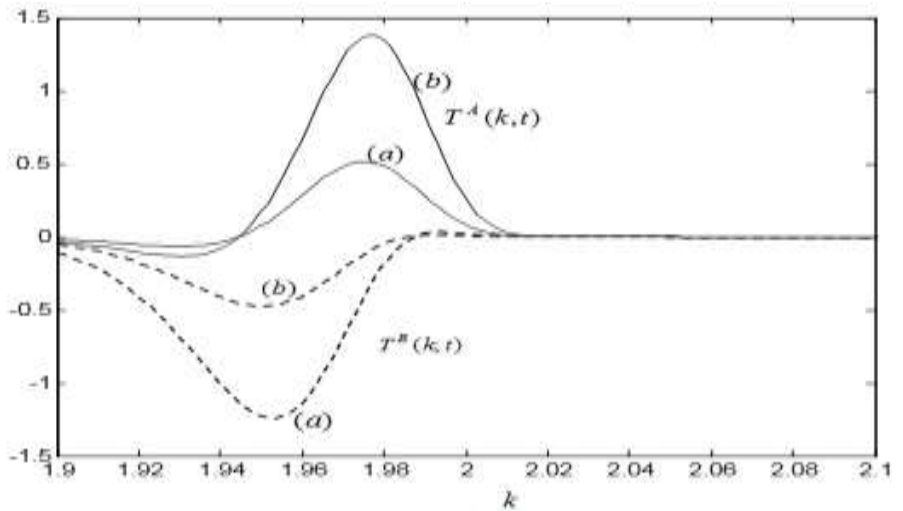

Figure 5.3 Energy Transfer Function of the reactant A (solid line) and the reactant $B$ (dashed line) for keynold number $\mathrm{K}-10^{+}$at (a) $\mathrm{t}-\mathrm{O}$ and (b) $\mathrm{t}=\mathrm{s}$

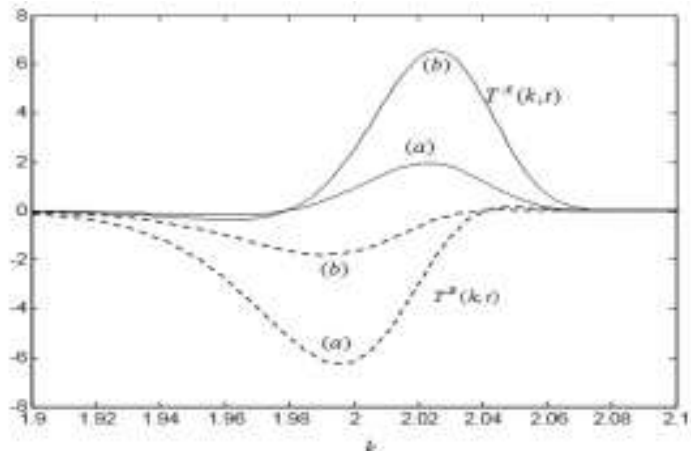

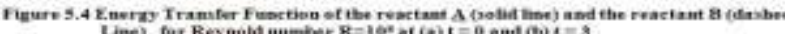

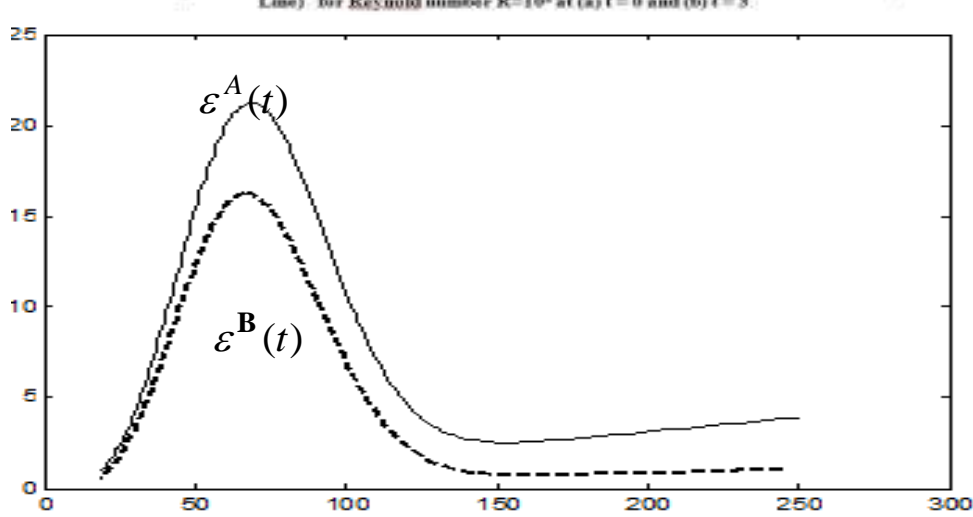

Figure 5.5 Enstropy of the reactant A (solid line) and the reactant B (dashed line) for $R=10^{4}$

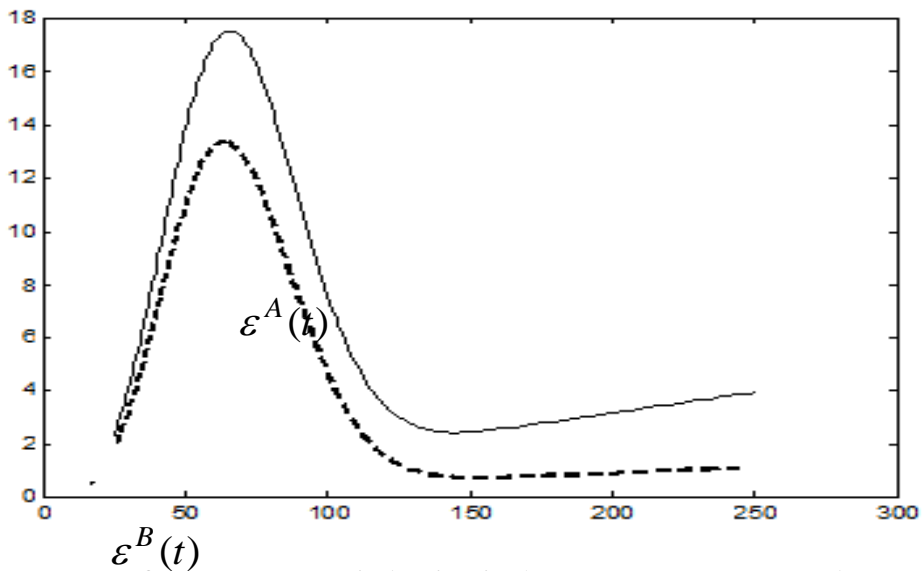

Figure 5.6 Enstropy of the reactant A (solid line) and the reactant B (dashed line) for Reynold number $-\ldots 6$ 


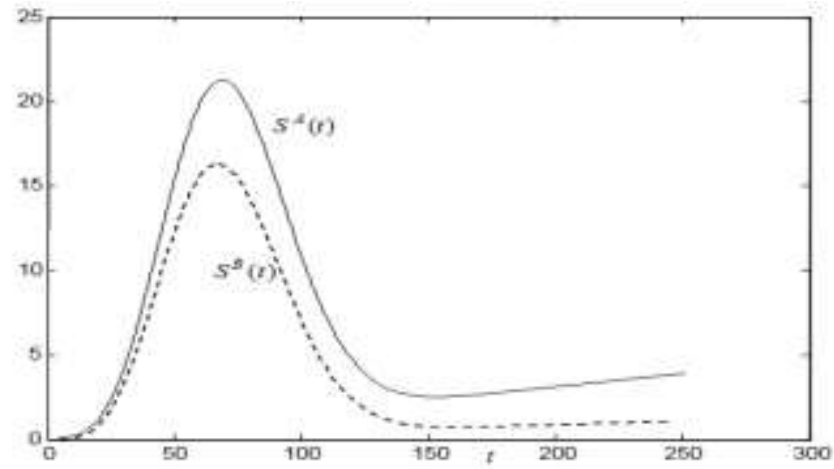

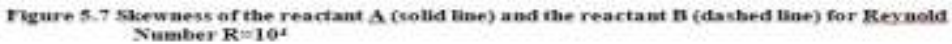

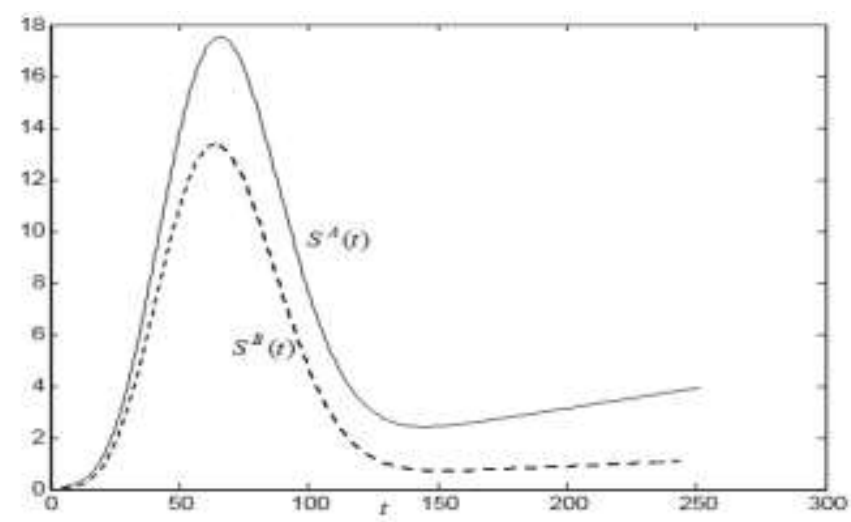

Figure 5.8 skewness of the reactant $\mathbf{A}$ (solsd tine) and the reactant $B$ (dashed line). for Reyneld Number $\mathbf{R}=10^{\circ}$

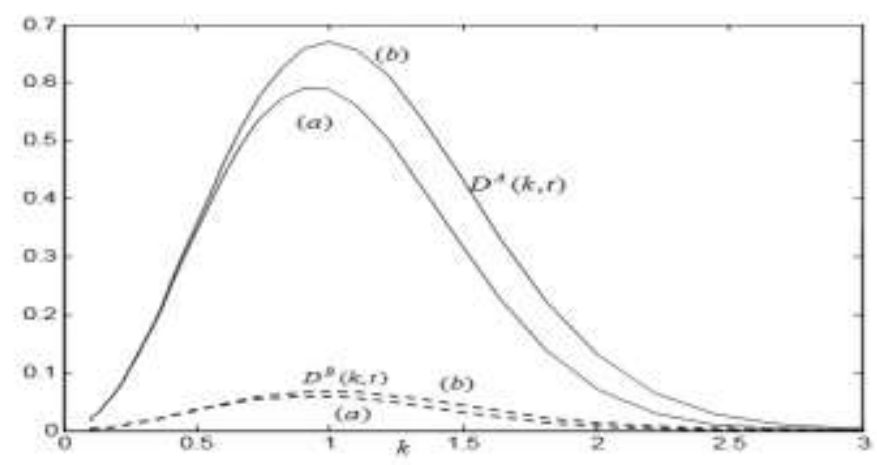

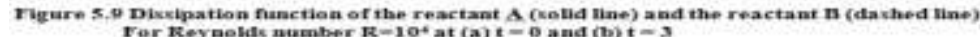

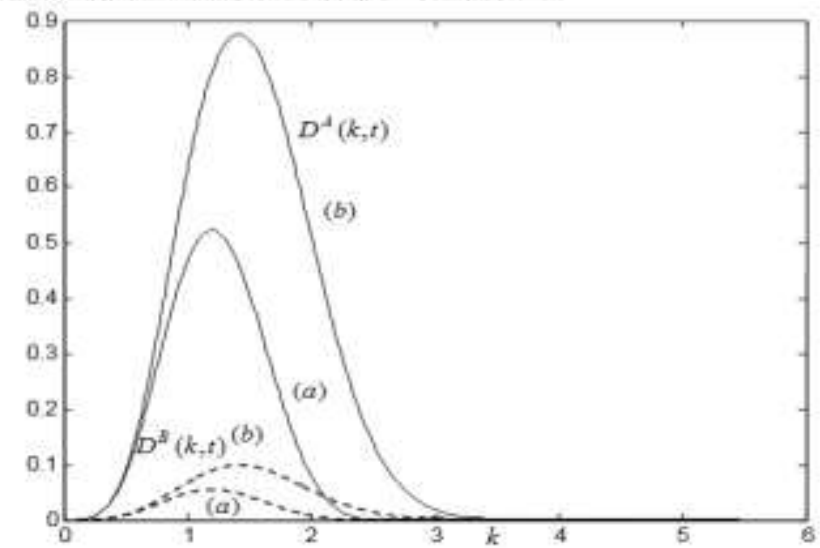

Figure 5.10 Dissipathon function of the reactant A (solid liee) and the reactant B (dashed line) for Reynold number $\mathrm{R}-10^{*}$ at $(\mathrm{a}) \mathrm{t}=0$ and (b) $t=3$ 

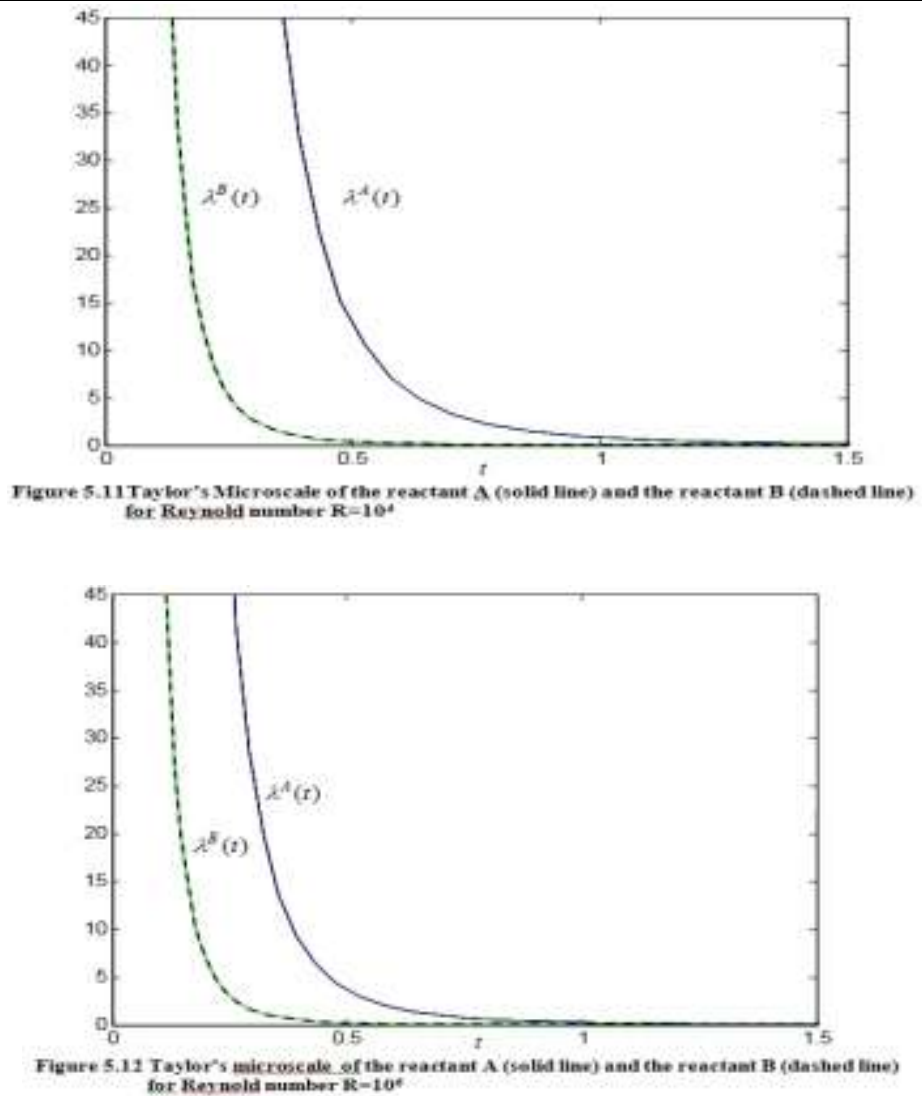

VI.

Summery And Discussion

We first write the equations for turbulent multi-component mixture (n-reactants) with second order, irreversible, isothermic reaction. In the present study we restrict ourselves to two reactants A and B. Joshi N.E. and Meshram M. C. ${ }^{5}$ investigated turbulence with reacting and mixing chemical elements of the type $\mathrm{A}+\mathrm{B} \rightarrow$ Product on employing the multiple-scale-cumulant-expansion method. The choice of multiple time scale and multiple length scale of Joshi N.E. and Meshram M. C. ${ }^{5}$ is retained in the present work. In order to improve the drawbacks of multiple-scale-cumulant-expansion method Tatsumi T. and Kida S. ${ }^{17}$ have introduced the modified-zero-fourth-order-cumulant-expansion method for investigation of homogeneous isotropic turbulence. We have employed this method for the investigation of turbulence with reacting and mixing chemical elements of the type $\mathrm{A}+\mathrm{B} \rightarrow$ Product. We consider the equations for the energy spectrum function for the specie $\mathrm{A}$ and that for the specie B derived by Joshi N.E. and Meshram M. C. ${ }^{5}$ on employing this method. These equations are then integrated numerically and various statistical quantities describing the turbulence with reacting and mixing chemical elements of the type $\mathrm{A}+\mathrm{B} \rightarrow$ Product such as energy of concentration fields, enstropy of the concentration fields, skewness of concentration fields, dissipation energy of concentration fields, transfer functions of concentration fields and Taylor's micro-scales for concentration fields A and B are evaluated and representative values for Reynolds numbers $\mathrm{R}=10^{4}$ and $\mathrm{R}=10^{6}$ are presented in the form of the graphs. The characteristics of turbulence with reacting and mixing elements reflected in these graphs are studied. The important features of the statistical quantities describing the turbulence under study reveal the following:

1. The energy spectrum function for both the reactant A and the reactant B are found to be positive definite for all values of Reynolds numbers.

2. The spectrum of the concentration fields extends much further into the high wave number range than that of the velocity fields.

3. The energy spectrum functions of reactant $A$ and that of $B$ is proportional to the negative $5 / 3$ power of the wave number for all the time in the inertial subrange whose extent increases indefinitely with Reynolds number. This result is in accordance with the Kolomogorov's second hypothesis.

4. The universal spectrums are composed of three different subspectra, which are in order of increasing wavenumber, the $\mathrm{k}^{-5 / 3}$ spectrum, the $\mathrm{k}^{-1}$ spectrum and the exponential spectrum.

5. The nature of the transfer spectra of both the chemical reactants A and B is analogous. As the Reynolds number takes the larger value the wavenumber at which $T^{A}(k, t)$ and $T^{B}(k, t)$ vanish is shifted to the lower wavenumber. 
6. The graphs of the enstropy for reactant A and B are analogous to each other. As the Reynolds number increases there is increase in the time taken to attain the maximum enstropy in case of both the reactants A and B.

7. Enstrophy of both the reactants grows very rapidly in the initial period and attains a quite large maximum value 21.5 for $\mathrm{R}=10^{4}$ and 16.5 corresponding to $\mathrm{R}=10^{6}$ for the reactant $\mathrm{A}$ respectively. Similarly the corresponding maximum values for the reactant $\mathrm{B}$ are 17.5 and 13.5 respectively.

8. In the initial period the enstropy of the specie A is proportional to second power of time and analogues to this enstropy of the specie B is proportional to 2.25 power of time. In the intermediate period The enstropy of the concentration field $\mathrm{A}$ is proportional to the negative 2.1 power of time and this law for the skewness of the concentration field $\mathrm{B}$ is proportional to the negative 2.13 power of time whereas in the third period the enstropy of the concentration field $\mathrm{A}$ is proportional to the 1.13 power of time and the enstropy of the concentration field $\mathrm{B}$ is proportional to the 1.03 power of time.

9. The initial value of skeness is identically zero for both the reactants.

10. The maximum value of the skewness attained by the reactant $A$ for $R=10^{4}$ is 21.5 and that for the reactant $B$ is nearly 16.5 . Similarly for $\mathrm{R}=10^{6}$ the maximum value of the skewness attained by the reactant $\mathrm{A}$ and reactant $\mathrm{B}$ is 17.5 and 13.5 approximately.

11. The graph of the skewness for both the reactants is divided into three different time period. In the initial period the skewness of the specie A is proportional to second power of time and analogues to this skewness of the specie B is proportional to 2.25 power of time. In the intermediate period the skewness of both the reactant assumes the Gaussian shape and the skewness of the concentration field A is proportional to the negative 2.1 power of time and the skewness of the concentration field $\mathrm{B}$ is proportional to the negative 2.13 power of time and finally in the third period skewness of the concentration field $\mathrm{A}$ is proportional to the 1.13 power of time and the skewness of the concentration field $\mathrm{B}$ is proportional to the 1.03 power of time.

12.In the energy containing range and the inertial subrange, as Reynolds number increases the energy dissipation decreasess in magnitude than the energy transfer.

13. An eminent feature obtained related to dissipation function is that for large Reynolds number the $\mathrm{k}^{-1}$ spectrum always occurs in the neighbourhood of wavenumber at which energy transfer changes its sign.

14. As the Reynolds number increases the time taken for obtaining zero Taylor's micro-scale for both the concentration fields A and B decreases.

15. The Taylor's micro-scale for both the reactants A and B is inversely proportional to half power of time.

The present investigations for turbulence with reacting and mixing chemical elements $\mathrm{A}+\mathrm{B} \rightarrow$ Product using modified-zerofourth-order-cumulant-expansion-approximation method has provided significant structure of turbulence under study. Therefore, we foresee that this method when employed for the investigation of similar turbulent flows may provide significant information in general and in the study of statistical investigation of turbulence with reacting and mixing chemical elements $\mathrm{A}+\mathrm{A} \rightarrow$ Product; atmospheric turbulence with reacting and mixing chemical elements in particular. The work in such areas is in progress and update shall be presented in due course of time.

[1] Brodkey R . S. (1975) Turbulence in Mixing Operations: Theory and Applications to Mixing and Reaction, New York: Academic Press.

[2] O'Brien E. E. (1971) Turbulent Mixing of Two Rapidly Chemical Species, Phys. Fluids 14, pp. 1326-1330.

[3] Lewis R. M. and Kraichnan R. H. (1962) A Space-Time Functional Formalism for Turbulence, Comm. Pure Appl. Math. 15,pp.397-411

[4] Hopf E. (1952) Statistical Hydrodynamics and Functional Calculus, J. Rat.Mech. Anal., 1, pp. 87-123.

[5] Joshi N. E. and Meshram M. C. (1988) The space-time functional formalism for turbulent multicomponent mixture with second order reactions, J.Math. Phy.Sci. 22(6), pp.711-737.

[6] Rosner D. (2000) Transport Processes in Chemically Reacting Flow, Dover, New York.

[7] Peters H. and Bokhorst R. (2000) Theoretical and Numerical Combustion, (R.T. Adverts Inc.).

[8] Poinsot and Veynante (2001) Theoretical and Numerical Combustion, (R.T. Adverts Inc.).

[9] Veynante and Vervisch (2002) Turbulent Combustion Modelling, Progressing Engg. Sci., 28, pp. 193-266.

[10] Meshram M.C. and Junghare J.R., 2004, Investigation of atmospheric turbulence with mixing and reacting elements of the type

[11] $\quad \mathrm{A}+\mathrm{B} \rightarrow$ Product using two-point closure method, Nagpur university Science Journal,11,pp.24

[12] Meshram M.C. and Junghare J.R., 2004, Investigation of atmospheric turbulence with mixing and reacting elements of the type

[13] $\quad \mathrm{A}+\mathrm{A} \rightarrow$ Product using two-point closure method, Nagpur university Science Journal,11,pp.09

[14] Meshram M.C. (2010) Two-point Closure Method for Turbulence with Reacting and Mixing Chemical Elements of type A+B $\rightarrow$ C, [15] Phys. Scr.T142, pp.1-8.

[16] Meshram M.C. (2012) Factorised Cumulant Expansion Approximation Method for Turbulence with Reacting and Mixing Chemical Elements of type A+B $\rightarrow$ Product, Phys. Scr.(To appear).

[17] Meshram M.C. and Sahu K.(2012) Investigation of turbulence with reacting and mixing chemical elements of the type A+B $\rightarrow$ Product described by space-time functional formalism, Int.Jour.Math.Sci. and Engg.Appl.(To appear)

[18] Frisch U., Lesieur M., Schertzer D. (1980) Comments on the Quasi-Normal Markovian Approximation for fully-developed turbulence, J. Fluid Mech., 97, p. 181-192.

[19] Tatsumi T., Kida S. and Mizushima J. (1978) The Multiple-Scale -Cumulant- Expansion for Isotropic Turbulence, J. fluid Mech., 85 , pp. 97-142.

[20] Tatsumi T. and Kida S. (1980) The Modified Cumulant Expansion forIsotropic Turbulence at Large Reynolds Numbers, J. Phys Soc. Japan, 49, pp. 2014-2025.

[21] Meshram M. C and Sahu K. (2012) Investigation of Magnetohydrodynamic Turbulence Described by the Space-Time Functional [22] Formalism, Physics Scripta (To appear). 